\title{
SINGULAR MEASURES AND THE KEY OF $G$
}

\author{
Stephen M. Buckley and Paul Macmanus
}

Abstract

We construct a sequence of doubling measures, whose doubling constants tend to 1 , all for which kill a $G_{\delta}$ set of full Lebesgue measure.

\section{Introduction}

A non-zero Borel measure $\nu$ is said to be doubling if there is a constant $C \geq 1$ such that

$$
C^{-1} \leq \frac{\nu(I)}{\nu(J)} \leq C,
$$

whenever $I, J$ are adjacent intervals of the same length. We call the smallest $C=C_{\nu}$ for which this condition holds, the doubling constant of $\nu$. A measure is a multiple of Lebesgue measure if and only if its doubling constant is 1 .

It was shown in $[\mathbf{B H M}]$ that if $U \subset[0,1]^{n}$ is open and $|\partial U|=0$, then $\nu_{n}(U) \rightarrow|U|$ whenever $\nu_{n}$ is a sequence of probability measures on $[0,1]^{n}$ whose doubling constants tend to 1 . In particular, if $U$ is an open subset of $[0,1]$ of full measure, then $\nu_{n}(U) \rightarrow 1$. We will show, amongst other things, that there exists a $G_{\delta}$ set $G$ in $[0,1]$ of full measure, and a sequence $\nu_{n}$ of measures whose doubling constants tend to 1 , yet $\nu_{n}(G)=$ 0 for all $n$. We can even choose the measures to be "renormalizations" of a single measure $\nu$ which "fit the gaps in $G$ " as a key fits a lock.

We wish to thank the referee for drawing our attention to the paper of Kakutani.

\section{Definitions and basic results}

There is an easy way, essentially due to Kahane $[\mathbf{K h}]$, to generate doubling measures. Let $\mathcal{Q}$ consist of all intervals on $[0,1)$ of the

2000 Mathematics Subject Classification. 60G30.

The first author was partially supported by Enterprise Ireland. 
form $\left[m 4^{-k},(m+1) 4^{-k}\right)$, where $m, k$ are non-negative integers, and set $\mathcal{Q}(j)$ to be the subset of $\mathcal{Q}$ consisting of those intervals of length $4^{-j}$.

For any $I \in Q$ the four children are labeled $I_{0}, I_{1}, I_{2}, I_{3}$, moving from left to right. Now consider

$$
H_{I}(x)= \begin{cases}1, & x \in I_{1} \\ -1, & x \in I_{2} \\ 0, & \text { otherwise. }\end{cases}
$$

The product $\prod_{I \in \mathcal{Q}}\left(1+a_{I} H_{I}\right)$ converges weak-* to a doubling, probability measure $\mu$, provided that $\sup _{I \in \mathcal{Q}}\left|a_{I}\right|<1$. We call any such measure $\mu$ a Kahane measure and write $\|\mu\|_{K}=\sup _{I \in \mathcal{Q}}\left|a_{I}\right|$. Furthermore, the doubling constant $C_{\mu}$ tends to 1 as $\|\mu\|_{K}$ tends to 0 ; in fact, if $\|\mu\|_{K} \leq$ $1-\epsilon$, then there is a constant $c_{\epsilon}$, dependent only on $\epsilon$, such that $C_{\mu} \leq$ $1+c_{\epsilon}\|\mu\|_{K}$ whenever for some $\epsilon>0$.

For our purposes it will be sufficient to consider Kahane measures for which all of the coefficients $a_{I}$ at any given scale are equal and $\|\mu\|_{K} \leq$ $1-\epsilon$ for some $\epsilon>0$, which we assume to be fixed from now on. We denote this class of measures by $\mathcal{M}_{\epsilon}$, or simply $\mathcal{M}$. Then every measure in $\mathcal{M}$ is of the form $\prod_{j=1}^{\infty}\left(1+a_{j} R_{j}\right)$ where $R_{j}=\sum_{I \in Q(j)} H_{I}$; it is convenient to introduce the notation $c_{j}(\mu) \equiv a_{j}$. We will focus on those measures $\mu \in \mathcal{M}$ for which $c_{j}(\mu) \rightarrow 0$ as $j \rightarrow \infty$, and we label these $\mathcal{M}_{0}$. For $\mu \in \mathcal{M}$ and $n=0,1,2, \ldots$ the measure $\mu_{n} \in \mathcal{M}$ henceforth denotes the element of $\mathcal{M}$ with $c_{j}\left(\mu_{n}\right)=c_{j+n}(\mu), j \in \mathbb{N}$. The measures $\mu_{n}$ are "renormalized" versions of $\mu$; in fact, if $S \subset[0,1)$ is a measurable set and $f_{S}$ is the periodic function with period 1 whose restriction to $[0,1)$ is the characteristic function of $S$, then $\mu_{n}(S)=\int_{0}^{1} f_{S}\left(4^{n} t\right) d \mu(t)$. Given $\mu \in \mathcal{M}_{0}$, it follows from the estimate in the last paragraph that the sequence of doubling constants $\left(C_{\mu_{n}}\right)$ has limit 1 . Thus every $\mu \in \mathcal{M}_{0}$ is optimally doubling at small scales in the sense that $\nu=\mu$ satisfies (1) with $C=C_{\mu_{n}}$ whenever $I, J$ are adjacent intervals with $|I|=|J| \leq 4^{-n}$.

The following result is a special case of a result of Kakutani $[\mathbf{K k}$, Corollary 1].

Theorem A. Let $\mu, \nu \in \mathcal{M}$, with $a_{j}=c_{j}(\mu), b_{j}=c_{j}(\nu)$, for all $j \in \mathbb{N}$. If $\left(a_{j}-b_{j}\right)_{j=1}^{\infty}$ lies in $l^{2}$, the class of square summable sequences, then $\mu \ll \nu \ll \mu$, otherwise $\mu \perp \nu$. 
In fact, when $\nu$ is Lebesgue measure and $\left(a_{n}\right) \in l^{2}$ above, more is true: $\mu$ lies in the Muckenhoupt class $A_{\infty}$, and in particular $\mu$ has density lying in $L^{p}([0,1])$ for some $p>1$; see $[\mathbf{B u}]$ and $[\mathbf{F K P}] .^{1}$

Kakutani proves this result by careful analysis, but let us pause to prove the singularity part of this result using the Lyapunov version of the Central Limit Theorem [Bi, Theorem 27.3] which we now state.

Theorem B. Suppose that $\left\{X_{n}\right\}_{n=1}^{\infty}$ is a sequence of independent random variables, and that the moments $E\left(X_{n}\right)=e_{n}, E\left(X_{n}-e_{n}\right)^{2}=\sigma_{n}^{2} \neq$ 0 , and $E\left|X_{n}-e_{n}\right|^{3}=\tau_{n}^{3}$ are finite for each $n$. Let

$$
s_{n}=\left(\sum_{i=1}^{n} \sigma_{i}^{2}\right)^{1 / 2}, \quad t_{n}=\left(\sum_{i=1}^{n} \tau_{i}^{3}\right)^{1 / 3} .
$$

If $\lim _{n \rightarrow \infty} t_{n} / s_{n}=0$, then $Y_{n} \equiv \sum_{i=1}^{n}\left(X_{i}-e_{i}\right) / s_{n}$ converges in distribution to the standard normal distribution.

In this paragraph we employ the notation of Theorem A. The functions $R_{n}$ are independent as random variables on $[0,1]$ with respect to $\nu$, and so the functions $f_{n}=\log \left[\left(1+a_{n} R_{n}\right) /\left(1+b_{n} R_{n}\right)\right]$ are also independent. A little calculation with the power series expansion for $\log (1+t)$ gives

$$
\begin{gathered}
E_{\nu}\left(f_{n}\right) \equiv e_{n}=-\frac{\left(a_{n}-b_{n}\right)^{2}}{4\left(1-b_{n}^{2}\right)}+O\left(\left|a_{n}-b_{n}\right|^{3}\right) \\
E_{\nu}\left(f_{n}-e_{n}\right)^{2} \equiv \sigma_{n}^{2}=\frac{\left(a_{n}-b_{n}\right)^{2}}{2\left(1-b_{n}^{2}\right)}+O\left(\left|a_{n}-b_{n}\right|^{3}\right) \\
E_{\nu}\left|f_{n}-e_{n}\right|^{3} \equiv \tau_{n}^{3}=\frac{\left|a_{n}-b_{n}\right|^{3}}{2} \frac{1+b_{n}^{2}}{\left(1-b_{n}^{2}\right)^{2}}+O\left(\left|a_{n}-b_{n}\right|^{4}\right) .
\end{gathered}
$$

Thus if $s_{n}, t_{n}$ are as in Theorem B, $\lim _{n \rightarrow \infty}\left|a_{n}-b_{n}\right|=0$, and $\left(a_{n}-\right.$ $\left.b_{n}\right)_{n=1}^{\infty} \notin l^{2}$, then $t_{n}^{3} / \sum_{i=1}^{n}\left|a_{i}-b_{i}\right|^{3}$ and $s_{n}^{2} / \sum_{i=1}^{n}\left(a_{i}-b_{i}\right)^{2}$ are bounded above and below by positive, finite constants that are independent of $n$. It is then routine to deduce that $\lim _{n \rightarrow \infty} t_{n} / s_{n}=0$; one simply splits the sum at a point beyond which $\left|a_{n}-b_{n}\right|$ is very small and uses the estimate $\|\cdot\|_{l^{3}} \leq\|\cdot\|_{l^{2}}^{2 / 3}\|\cdot\|_{l^{\infty}}^{1 / 3}$. Thus Theorem B is applicable in the case $X_{n}=f_{n}$. Since $\sum_{i=1}^{n} e_{i}$ is much larger than $s_{n}$ for large $n$, it follows that $Y_{n}$ tends to $-\infty$ in $\nu$-measure and thus $\prod_{n=1}^{\infty}\left(1+a_{n} R_{n}\right) /\left(1+b_{n} R_{n}\right)$ converges in $\nu$-measure to the zero function. Set $\left\{P_{N}\right\}$ to be the partial products of this infinite product. We have

\footnotetext{
${ }^{1}$ These references only say that $\mu$ lies in dyadic $A_{\infty}$ but, since $\mu$ is a doubling measure, this implies that $\mu \in A_{\infty}$.
} 
just seen that this sequence of functions converges to zero in $\nu$-measure. However, $P_{N}(x)=\mu\left(I_{N}(x)\right) / \nu\left(I_{N}(x)\right)$, where $I_{N}(x)$ is the unique element of $\mathcal{Q}(N)$ containing $x$ and so, by the Radon-Nikodym theorem, $\left\{P_{N}\right\}$ converges $\nu$-a.e. to the Radon-Nikodym derivative of $\mu$ with respect to $\nu$. Consequently, the Radon-Nikodym derivative is zero $\nu$-a.e., and so $\mu \perp \nu$ whenever $\left(a_{j}-b_{j}\right) \notin l^{2}$.

We are mainly interested in Theorem A when $\nu$ is Lebesgue measure. In this case if the sequence $\left(c_{j}(\mu)\right)$ has limit zero but does not lie in $l^{2}$, then $\mu$ is a singular measure which is optimal doubling at small scales. The mere existence of such a measure may seem a little surprising and was only recently established (using different techniques) by Cantón $[\mathbf{C}]$ and Smith $[\mathbf{S}]$.

There is an obvious bijection, $A$, between $\mathcal{Q}$ and the set of finite sequences whose terms lie in $\{0,1,2,3\}$. We will refer to $A(I)$ as the address of $I$. The $j$ th term in the address is $A_{j}(I)$. For $I \in Q$, we let $E(I)$ consist of the union of the intervals $J \in \mathcal{Q}$ for which $A_{2 j}(J)=A_{j}(I)$ for all $j$. So the odd terms in $A(J)$ are arbitrary and the even terms are specified. If $I \in \mathcal{Q}(j), E(I)$ consists of $4^{j}$ elements of $\mathcal{Q}(2 j)$. For $n=0,1,2, \ldots$ and $I \in Q, T_{n}(I)$ consists of those intervals $J \in \mathcal{Q}$ for which $A_{n+j}(J)=A_{j}(I)$ for all $j$. So the first $n$ terms of $J$ are arbitrary and the remainder are specified. When $I \in \mathcal{Q}(j), T_{n}(I)$ consists of $4^{n}$ elements of $\mathcal{Q}(j+n)$. Note that if $I$ and $J$ are disjoint, then $E(I)$ and $E(J)$ are disjoint, as are $T_{n}(I)$ and $T_{n}(J)$. For any set $B$ that is a union of disjoint elements $I$ of $\mathcal{Q}$, we define $E(B)$ to be the union of the $E(I)$, and we define $T_{n}(B)$ similarly. It is easy to check that $|E(B)|=|B|$ and that $\left|T_{n}(B)\right|=|B|$.

Let $\Sigma_{j}$ be the collection of subsets of $[0,1)$ that are unions of elements of $Q(j)$. Any set $B \in \Sigma_{m}$ is said to be $j$-indifferent if whenever $B \supset I \in$ $Q(m)$ and $J$ is one of the three elements of $Q(m)$ for which $A(J)$ and $A(I)$ differ only in the $j$ th place, then $J \subset B$. Equivalently if $S(B)$ is the set of sequences of length $m$ given by $A(I)$ for each $I \in \mathcal{Q}(m), I \subset B$, then $B$ is $j$-indifferent precisely if $S(B)$ is measurable with respect to the $\sigma$-algebra generated by the sets

$$
S_{k, l}=\left\{\left(a_{i}\right)_{i=1}^{m}: a_{k}=l\right\}, \quad 1 \leq k \leq m, k \neq j, l \in\{0,1,2,3\} .
$$

The point of this definition is that if $B$ is $j$-indifferent, then $\mu(B)$ does not depend on the $c_{j}(\mu)$. In particular, if $B \in \Sigma_{m}$, then $E(B)$ is $j$-indifferent for all odd numbers $j$ and all even $j>2 m$, and $T_{n}(B)$ is $j$-indifferent for all $j \leq n$ and all $j>n+m$. 


\section{Construction of $\mu$ and $G$}

Our main result is as follows.

Theorem 1. There exists a measure $\mu \in \mathcal{M}_{0}$ on the interval $[0,1)$ and $a G_{\delta}$ set $G$ contained in $[0,1)$ which have the following properties:

(a) $\mu([0,1))=1,|G|=1$ and $\mu(G)=0$.

(b) $\mu_{n}(G)=1$ for all odd $n \in \mathbb{N}$ and $\mu_{n}(G)=0$ for all even $n \in \mathbb{N}$.

Taking $\nu_{n}=\mu_{2 n}$, we immediately get

Corollary 2. There exists a $G_{\delta}$ set $G$ in $[0,1]$ of full measure and a sequence $\nu_{n}$ of probability measures on $[0,1]$ whose doubling constants tend to 1 and for which $\nu_{n}(G)=0$ for all $n$.

The oscillatory behaviour of $\mu_{n}(G)$ described in Theorem 1(b) is all the more remarkable since the measures $\mu_{n}$ are renormalized versions of a single measure $\mu$ whose doubling constants are tending to one. The idea is to construct $G$ from sets that are indifferent at odd levels $n$ (and thus treat such $\mu_{n}$ like Lebesgue measure), but which are concentrated in areas where $\mu_{n}$ is small whenever $n$ is even.

Proof of Theorem 1: Let $b$ be any number strictly between 0 and 1. Define $\nu_{k}$ to be the element of $\mathcal{M}$ whose coefficients are all $2^{-k}$. This measure is singular with respect to Lebesgue measure. It follows that for sufficiently large $n_{k}$, there exists $A_{k} \in \Sigma_{n_{k}}$ for which $\left|A_{k}\right| \geq 1-b^{k}$ and $\nu_{k}\left(A_{k}\right) \leq b^{k}$. We can assume that the $n_{k}$ are increasing to $\infty$.

Divide the natural numbers into consecutive blocks $B_{1}, B_{2}, \ldots$ of length $2 n_{1}, 2 n_{2}, \ldots$. Set $a_{j}=2^{-k}$ whenever $j$ is an even number in block $B_{k}$, and 0 otherwise. Define $\mu \in \mathcal{M}_{0}$ by the equations $c_{j}(\mu)=a_{j}$.

Now let $m_{k}=2 n_{1}+\cdots+2 n_{k-1}$ for $k>1$ and $m_{1}=0$. Thus $m_{k}$ is the total length of the blocks $B_{1}, \ldots, B_{k-1}$. Define $H_{k}$ to be $T_{m_{k}}\left(E\left(A_{k}\right)\right)$. Then $H_{k} \in \Sigma_{m_{k}+2 n_{k}}$ and is $j$-indifferent for all $j$ except even numbers larger than $m_{k}$ and no larger than $m_{k}+2 n_{k}$, i.e., all even numbers in $B_{k}$. Remove the endpoints of the intervals that make up $H_{k}$ to get an open set $U_{k}$. The sets $U_{k}$ and $H_{k}$ differ only by a countable number of points. Thus any doubling measure gives them the same measure (doubling measures on the line are non-atomic). Set $G_{m}=\bigcup_{k=m}^{\infty} U_{k}$ and $G=\bigcap_{m=1}^{\infty} G_{m}$. This set $G$ is a $G_{\delta}$ set.

We have $\left|H_{k}\right|=\left|A_{k}\right| \geq 1-b^{k}$ for all $k$, hence $\left|G_{m}\right|=1$ for all $m$, and $|G|=1$. If $n$ is odd and $j$ is even, then $c_{j}\left(\mu_{n}\right)=0$. But $H_{k}$ is $j$-indifferent for all odd $j$, so it follows that $\mu_{n}\left(H_{k}\right)=\left|H_{k}\right|$. As a result, $\mu_{n}(G)=1$ whenever $n$ is odd. 
The set $H_{k}$ is $j$-indifferent for all $j$ except even $j$ in $B_{k}$ and $c_{j}(\mu)=$ $2^{-k}$ for these exceptional integers. Thus $\mu\left(H_{k}\right)=\nu_{k}\left(A_{k}\right) \leq b^{k}$. Consequently, $\mu\left(G_{m}\right) \leq b^{m}(1-b)^{-1}$ for all $m$, and so $\mu(G)=0$.

Suppose $n-m$ is even. Then $c_{j}\left(\mu_{n}\right)=c_{j}\left(\mu_{m}\right)$ for "most" values of $j$ in the sense that for each $k$ the number of places where the coefficients of size $2^{-k}$ do not match up is bounded independently of $k$, indeed by $n-m$. It follows readily from Theorem A that $\mu_{n} \ll \mu_{m} \ll \mu_{n}$. In particular, $\mu_{n}(G)=0$ for all even $n$.

Finally, we note two facts about the relationship between $\mu_{n}$ and $\mu_{m}$. First, if $n-m$ is odd, then one of $n, m$ is odd and the other is even. Thus one of the measures gives full measure to $G$, while the other gives $G$ zero measure. In particular, $\mu_{n} \perp \mu_{m}$. Secondly, when $n-m$ is even, the absolute continuity mentioned in the last paragraph of the proof can be strengthened: there exists a constant $C$, dependent only on $n-m$, such that $C^{-1} \mu_{m}(E) \leq \mu_{n}(E) \leq C \mu_{m}(E)$. It suffices to prove this last estimate for $E \in \mathcal{Q}$, in which case the estimate follows from the fact, that $c_{j}\left(\mu_{n}\right)=c_{j}\left(\mu_{m}\right)$ for "most" values of $j$. We leave the details to the reader.

\section{References}

[Bi] P. Billingsley, "Probability and measure", third ed., Wiley Series in Probability and Mathematical Statistics. A WileyInterscience Publication, John Wiley \& Sons Inc., New York, 1995.

[Bu] S. M. BuCKLEY, Estimates for operator norms on weighted spaces and reverse Jensen inequalities, Trans. Amer. Math. Soc. 340(1) (1993), 253-272.

[BHM] S. M. Buckley, B. Hanson and P. Macmanus, Doubling for general sets, Math. Scand. (to appear).

[C] A. Cantón, Singular measures and the little Bloch space, Publ. Mat. 42(1) (1998), 211-222.

[FKP] R. A. Fefferman, C. E. Kenig and J. Pipher, The theory of weights and the Dirichlet problem for elliptic equations, Ann. Math. (2) 134(1) (1991), 65-124.

[Kh] J.-P. Kahane, Trois notes sur le ensembles parfaits linéaires, Enseignement Math. (2) 15 (1969), 185-192.

[Kk] S. KaKUtani, On equivalence of infinite product measures, Ann. of Math. (2) 49 (1948), 214-224. 
[S] W. Smith, Inner functions in the hyperbolic little Bloch class, Michigan Math. J. 45(1) (1998), 103-114.

Department of Mathematics

National University of Ireland

Maynooth, Co. Kildare

Ireland

E-mail address: sbuckley@maths.may.ie

E-mail address: pmm@maths.may.ie

Primera versió rebuda el 26 d'octubre de 1999, darrera versió rebuda el 19 de juny de 2000. 